\title{
Use of a monoclonal antibody and a DNA probe for diagnosing acute toxoplasmosis in ambiguous
} cases

\author{
J C Garberi, S O Angel, P Paulin, V Pszenny, L Romano
}

\begin{abstract}
An unusual case of cerebral toxoplasmosis in an HIV negative 11 year old child is reported. Central nervous system disease was assessed immunohistochemically in a brain biopsy specimen with TgP8-a specific monoclonal antibody against Toxoplasma gondii antigens-thus confirming IgG and IgM serology, technetium scan findings, and clinical data. In addition, an active parasitaemia was confirmed by DNA in situ hybridisation assay in white cells using an ABGTg4 probe. The child recovered after specific $T$ gondii treatment. Follow up six months later showed that he was immunodeficient.
\end{abstract}

(F Clin Pathol 1994;47:853-854)

Toxoplasmosis is the most common cause of focal brain lesions in the immunocompromised, such as those with AIDS or transplant recipients. ${ }^{12}$ However, it is quite rare to find this condition in immunocompetent people in whom the course of the disease is usually harmless. Moreover, when macroscopic and microscopic signs of central nervous system disorders become evident in these patients, they are often misdiagnosed. The case reported here illustrates the fact that even in an apparently normal child, a clear case of cerebral toxoplasmosis was found.

\section{Case report}

A young, white, 11 year old boy, with no nutritional deficiency and previously healthy, was admitted to hospital. He had had a convulsive episode three months earlier. He had no history of epilepsy. The patient had a further convulsive episode with generalised tonic clonic convulsions and loss of consciousness. Therefore, anticonvulsive treatment was applied.

Routine laboratory analyses were normal: haematocrit of $40 \%$; total white cells $4.5 \times$ $10 \% / 1$. White cell differential was: polymorphonuclear cells $1.90 \times 10^{9} / 1$; lymphocytes $1.90 \times 10^{9} / 1$; monocytes $0.18 \times 10^{9} / 1$ and eosinophils $0.45 \times 10^{\%} / 1$. At that time CD4 and CD8 counts were $1.16 \times 10^{9} / 1$ and $0.43 \times 10^{9} / 1$, respectively. Serological tests for HIV 1 and HIV 2, syphilis and Chagas's disease were negative. At the time of admission, toxoplasma serology was not analysed because he had not presented with fever, lymphadenopathy, or any other symptom suspicious of toxoplasmosis. His electroencephalogram and retinal exams were normal.
However, a computed tomography brain scan showed a hypodense heterogenous lesion at the left side of the fronto-parietal region and a presumptive diagnosis of cerebral tumor was suggested. Lumbar puncture was not attempted because of raised intracranial pressure. In view of this, a brain biopsy was performed and samples taken. A diagnosis of cerebral toxoplasmosis was finally confirmed by serology, immunohistology, and DNA in situ hybridisation. $\mathrm{He}$ was given pyrimetamine, sulphadiazine, and folic acid. Two months later he was well enough to be discharged.

\section{Methods}

Specific total antibodies against $T$ gondii were determined using the dye test, and specific IgM antibodies by immunosorbent agglutinating assay (ISAGA).

TgP8, a monoclonal antibody against the $\mathrm{RH}$ strain of $T$ gondii tachyzoites, was produced in our laboratory. ${ }^{3}$ It is an IgM class immunoglobulin that recognises surface antigens of 43,55, and 66 kilodalton molecular weights.

Brain biopsy specimens were fixed in $10 \%$ buffered formaldehyde and embedded in paraffin wax using routine procedures. The tissue was stained with haematoxylin and eosin and immunostained with TgP8 monoclonal antibody using the peroxidase-anti-peroxidase technique.

Blood samples were obtained and both sera and buffy coat were separated by centrifugation. DNA was extracted by treatment with proteinase $\mathrm{K}$-sodium dodecyl sulphate and phenolchloroform. DNA hybridisation was performed with a non-radioactive ABGTg4, a $T$ gondii exclusive repetitive DNA sequence. ${ }^{4}$

The immunological response of the patient was monitored after he had been discharged. Six months later, total leucocyte count was $5.9 \times 10^{9} / 1$; the white cell differential was: polymorphonuclear cells $5 \cdot 19 \times 10^{9} / 1$; lymphocytes $0.52 \times 10^{9} / 1$; monocytes $0.06 \times 10^{9} / 1$; and eosinophils $0.06 \times 10^{\%} / 1$. CD4 counts decreased to $0.29 \times 10^{9} / 1$ and $\mathrm{CD} 8$ counts to $0 \cdot 21 \times 10^{9} / 1$. Results of HIV 1 and HIV 2 serotype analyses remained negative. Nonspecific mitogen lymphocyte transformation tests yielded normal results. However, in vivo reactions to cellular immunity stimulating antigens, such as tetanic toxoid or purified protein derivate (PPD), were all negative. There was only a slight improvement in response to some of these antigens after treatment with cellular immunity stimulating drugs. 


\section{Results}

Histopathological examination of brain tissue showed encephalic parenchyma with a diffuse and perivascular infiltrating inflammatory reaction. The inflammatory cells were mainly lymphoplasmocytic cells, macrophages and lymphocytes. This histological pattern strongly suggested an infectious disease.

At this time further blood samples were obtained and Toxoplasma specific antibodies were measured (dye test: 1/64000 and ISAGA: 1/64).

Immunohistochemistry was performed on brain slides, with TgP8. $T$ gondii tachyzoite free forms were obvious among inflammatory cells. Therefore, a diagnosis of cerebral toxoplasmosis was clearly established.

To investigate possible parasitaemia in similar cases, blood samples were collected. The presence of Toxoplasma DNA in blood samples was then sought using a DNA hybridisation assay with the digoxigenin labelled ABGTg4 DNA probe and specific $T$ gondii DNA was detected. DNA extracted from a healthy $T$ gondii seronegative individual and from an HIV positive patient with a confirmed case of cerebral toxoplasmosis were assayed at the same time as negative and positive controls, respectively. Samples were always tested in triplicate.

\section{Discussion}

Acquired toxoplasmosis is often a mild disease in immunocompetent people. Therefore, cerebral manifestations of this disease are commonly regarded as symptoms seen in immunocompromised patients. However, as early as 1941 Sabin et al had already described toxoplasmic meningoencephalitis in an apparently normal 6 year old boy. ${ }^{5}$ In the case reported here the patient showed a good humoral immune response and with the exception of a slight decrease in the numbers of peripheral white cells $\left(4.5 \times 10^{9} / 1\right)$, no other indicators pointed to immunosuppression at the time of presentation. However, six months later the patient was immunosuppressed with a decrease in numbers of total peripheral lymphocytes $(10 \%$ of total white cells), low number of CD4 lymphocytes, and no response to cellular immunity estimulating antigens. He was, however, HIV negative. This immunological response might explain why cerebral toxoplasmosis occurred. The aetiology of his depressed immunity is now being studied.

Thus when comparable clinical symptoms and computed tomogram findings are presented the possibility of central nervous system toxoplasmosis should be considered. In these cases accurate and rapid diagnosis is essential for patient management and prognosis, but this is not an easy task. Although serology is sometimes indicative of infection, it is not sufficient to establish an active and disseminated stage of the disease. On the other hand, we could not infer from serological titres how much the lesion had progressed. High titres of IgM and IgG anti-T gondii anti- bodies have also been described in patients with chronic toxoplasmosis. ${ }^{6}$ Therefore, up to now, conclusive diagnosis of cerebral toxoplasmosis hinges on the identification of trophozoites in the cerebrospinal fluid (when this is possible) and demonstration of the organism in cerebral biopsy specimens.

A simple haematoxylin and eosin stain is not enough for cerebral biopsy specimens, as owing to its small size, the organism has been confused with various other parasites. Porter and Sande reported a 16 to $22 \%$ prevalence of toxoplasmosis in patients with AIDS who had undetectable levels of antigen to Toxoplasma antibodies. ${ }^{7}$ In their study a patient was considered to have toxoplasmosis if there was histological proof of central nervous system infection, in addition to brain lesions on computed tomograms. TgP8, the monoclonal antibody used here, proved a very sensitive reagent, free of background interference, for immunohistochemical diagnosis of toxoplasmosis. Moreover, its specificity had been studied and confirmed in histopathological analyses performed in experimentally infected murine organs (data not shown).

The presence of parasites in blood samples is indicative of active infection. We have already described the detection of Toxoplasma DNA in blood samples from experimentally infected mice, ${ }^{4}$ and in the cerebrospinal fluid of HIV positive patients with cerebral toxoplasmosis, ${ }^{8}$ using a dot blot assay. Recently, the presence of parasites in human blood samples was shown using a nested polymerase chain reaction technique. ${ }^{9}$ In this study we were able to detect $T$ gondii in the peripheral leucocytes of the patient using a simple and rapid alternative technique. Further work is currently being carried out in our laboratory to assess the clinical importance of $T$ gondii DNA detection in blood samples using the ABGTg4 probe.

Finally, we presented two new tools for direct and specific detection of $T$ gondii in human samples. These should help to obtain an accurate diagnosis in ambiguous cases such as the one presented here.

This work was partially supported by Agencia Espanola de Cooperación Internacional-ICI, and by Fundación Alberto Roemmers.

1 Luft BJ, Remington JS. Toxoplasmic encephalitis. $\mathcal{f}$ Infect Dis 1988;157:1-13.

2 Brooks RG, Remington JS. Transplant related infections In: Benett JV, Brachman PS, eds. Hospital infections. Boston: Little, Brown and Company, 1986:581-618.

3 Angel SO, Pastini A, Pszenny V, et al. Monoclonal antibodies against Toxoplasma gondii for histopathological diagnosis. Medicina (Buenos Aires) 1991;51:85-6.

4 Blanco JC, Angel SO, Maero E, et al. Cloning of repetitive DNA sequences from Toxoplasma gondii and their usefulness for parasite detection. Am $f$ Trop Med Hyg fulness for paras

5 Sabin $\mathrm{A}$. Toxoplasma encephalitis in children. $\mathscr{f} A M A$ 1941;116:801-7.

6 Feldman HA, Miller LT. Serological study of toxoplasmosis prevalence. Am $\mathcal{F}$ Hyg 1956;64:320-35.

7 Porter SB, Sande MA. Toxoplasmosis of the central nervous system in the acquired immunodeficiency syndrome. N Engl $₹$ Med 1992;327:1643-8.

8 Angel SO, Maero E, Blanco JC, et al. Early diagnosis of toxoplasmic encephalitis in AIDS patients by dot blo hybridization analysis. $\mathcal{f}$ Clin Microbiol 1992;30:3286-7.

9 Hoyen DO, Joss AWL, Balfour AH, et al. Use of polymerase chain reaction to detect Toxoplasma gondii in human blood samples. $f$ Clin Pathol 1992;45:910-3. 